UNIVERSITÀ DI PISA

DIPARTIMENTO DI INFORMATICA

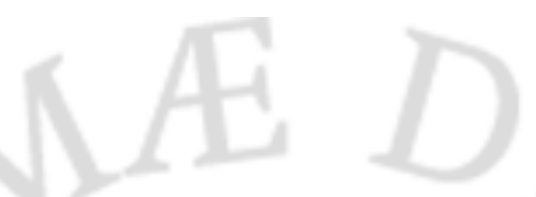

TECHNICAL REPORT

Solving Stochastic Hydrothermal Unit

Commitment with a New Primal Recovery

Technique Based on Lagrangian Solutions

Murilo Reolon Scuzziato, Erlon Cristian Finardi, Antonio Frangioni

July 20, 2020

LICENSE: Creative Commons: Attribution-Noncommercial - No Derivative Works ADDRESS: Largo B. Pontecorvo 3, 56127 Pisa, Italy - Tel. +39 050221270 



\title{
Solving Stochastic Hydrothermal Unit Com- mitment with a New Primal Recovery Tech- nique Based on Lagrangian Solutions
}

\author{
Murilo Reolon Scuzziato, Erlon Cristian Finardi, Antonio Frangioni
}

\begin{abstract}
The high penetration of intermittent renewable generation has prompted the development of Stochastic Hydrothermal Unit Commitment (SHUC) models, which are more difficult to be solved than their thermal-based counterparts due to hydro generation constraints and inflow uncertainties. This work presents a SHUC model applied in centralized cost-based dispatch, where the uncertainty is related to the water availability in reservoirs and demand. The SHUC is represented by a two-stage stochastic model, formulated as a large-scale mixed-binary linear programming problem. The solution strategy is divided into two steps, performed sequentially, with intercalated iterations to find the optimal generation schedule. The first step is the Lagrangian Relaxation (LR) approach. The second step is given by a Primal Recovery based on LR solutions and a heuristic based on Benders' Decomposition. Both steps benefit from each other, exchanging information over the iterative process. We assess our approach in terms of the quality of the solutions and running times on space and scenario LR decompositions. The results show the advantage of our primal recovery technique compared to solving the problem via MILP solver. This is true already for the deterministic case, and the advantage grows as the problem's size (number of plants and/or scenarios) does.
\end{abstract}

Index Terms - Stochastic Hydrothermal Unit Commitment, Lagrangian Relaxation, Primal Recovery Technique.

\section{NOMENCLATURE}

$N \quad$ number of nodes in the scenario tree;

$N S^{1} \quad$ number of nodes in the first stage of the scenario tree;

$p_{n} \quad$ probability of node $n$;

$I \quad$ number of thermal plants, such that $i=1, I$;

$I_{b} \quad$ number of thermal plants connected to bus $b$;

$R \quad$ number of hydro plants, such that $r=1, R$;

$R_{b} \quad$ number of hydro plants connected to bus $b$;

$C A \quad$ number of cascades, such that $c a=1, C A$;

$J_{r} \quad$ number of groups (with identical units) in the hydro $r$, with $j=1, \mathrm{~J}_{r}$;

$B$ number of buses in the power system, such that $b=1, B$;

tup $_{i}$, minimum uptime of the thermal plant $i$ (after start period) $[\mathrm{h}]$;

$t d o w n_{i}$ minimum downtime of the thermal plant $i$ (after shutdown period) $[\mathrm{h}]$;

$\Delta^{u p_{i}} \quad$ maximum ramp-up rate of the thermal plant $i[\mathrm{MW} / \mathrm{h}]$;

$\Delta^{\text {down }}{ }_{i}$ minimum ramp-down rate of the thermal plant $i$ $[\mathrm{MW} / \mathrm{h}]$;

$\Omega \quad$ number of scenarios, such that $\omega=1, \Omega$;

$\operatorname{Res}_{n} \quad$ spinning reserve requirement related to node $n$ [MW];

This work received partial financial support by $\mathrm{CNPq}$ and by the European Union Horizon 2020 research and innovation program under grant agreement No 773897 "plan4res".

M. R. Scuzziato is with Federal Institute of Santa Catarina, Florianópolis, Brazil (murilo.scuzziato@ifsc.edu.br). E. C. Finardi is with Federal University of Santa Catarina/LabPlan, Florianópolis, Brazil (erlon.finardi@ufsc.br) and INESC P\&D Brasil, Santos, Brazil. A. Frangioni is with Dipartimento di Informatica, Università di Pisa, Pisa, Italy (frangio@di.unipi.it).
$S C_{i} \quad$ startup cost of the thermal plant $i[\mathrm{R} \$]$;

$y_{r} \quad$ incremental inflow of the hydro plant $r\left[\mathrm{~m}^{3} / \mathrm{s}\right]$;

$v_{r}^{\text {target }}$ volume target of the hydro plant $r$ at the end of the horizon $\left[\mathrm{hm}^{3}\right]$;

$D_{b} \quad$ demand requirement at bus $b[\mathrm{MW}]$;

$\mathrm{fl}^{\max } \quad$ power flow limit of the line $l[\mathrm{MW}]$;

$\Gamma \quad$ power transfer distribution factor;

$\mu_{1} \quad$ weight parameter related to operational cost and proximal terms;

$\mu_{2} \quad$ weight parameter related to pseudo-schedule and LRsubproblems solution;

$\mu_{3} \quad$ weight parameter related to the proximal term between the continuous and binary variables.

ch index related to the piecewise linear approximation of the hydro production function;

$\mathrm{CH}_{j r} \quad$ set of linear approximations of the group $j$ in the hydro plant $r$;

cf index related to the linear approximation of the thermal cost function;

$C F_{i} \quad$ set of linear approximations related to the cost function of thermal plant $i$;

$n \quad$ index related to the nodes of the scenario tree;

$n-t \quad$ ascendant node related to the node $n$ at $t$ previous immediately periods in the same scenario;

$n+t \quad$ descendant node related to the node $n$ at $t$ periods forward in time in the same scenario;

$R_{r}^{+} \quad$ set of upstream reservoirs of the hydro plant $r$;

$R^{c a} \quad$ set of hydro plants in the cascade $c a$;

$N(\omega)$ set of all nodes of scenario $\omega$.

$p t_{\text {in }} \quad$ power generation of thermal plant $i$ at node $n$ [MW];

$u_{\text {in }} \quad$ binary variable that represents the commitment status of the thermal plant $i$ at node $n$;

up in binary variable that represents the startup status of thermal plant $i$ at node $n$;

$u d_{i n}$ binary variable that represents the shutdown status of thermal plant $i$ at node $n$;

$F_{\text {in }} \quad$ generation cost of thermal plant $i$ at node $n[\mathrm{R} \$]$;

$p h_{r n} \quad$ power generation of hydro plant $r$ at node $n[\mathrm{MW}]$;

$v_{r n} \quad$ volume of reservoir $r$ at node $n\left[\mathrm{hm}^{3}\right]$;

$d_{r n} \quad$ outflow of hydro $r$ at node $n$, i.e., the sum of turbined outflow and the spillage $\left[\mathrm{m}^{3} / \mathrm{s}\right]$;

$S_{r n} \quad$ spillage of hydro plant $r$ at node $n\left[\mathrm{~m}^{3} / \mathrm{s}\right]$;

$p h g_{j r n}$ power generation of the group $j$ in the hydro plant $r$ at node $n[\mathrm{MW}]$;

$q_{j r n} \quad$ turbined outflow of the group $j$ in the hydro plant $r$ at node $n\left[\mathrm{~m}^{3} / \mathrm{s}\right]$;

$z_{j r n} \quad$ binary variable that represents the commitment status of the group $j$ in the hydro plant $r$ at node $n$;

$f p g h_{j r n}$ hydro production function of the group $j$ and hydro plant $r$ at node $n[\mathrm{MW}]$ 
foptin cost function of the thermal plant $i$ at node $n$ [MW];

$o o f_{n} \quad$ total operating cost at node $n$;

$p x t^{P S}$ proximal term related to the pseudo-schedule;

$p x t^{L R} \quad$ proximal term related to the LR solutions;

$a f c_{n} \quad$ approximation of the future cost at node $n$;

$x_{n}, u_{n}$ continuous (binary) variables related to node $n$;

$\mathscr{X}_{n}, \ell_{n} \ell_{0}$ continuous (binary) variables associated with pseudoschedule at node $n$;

$\hat{x}_{n}, \hat{u}_{n}$ continuous (binary) variables associated with LRsubproblems at node $n$;

$\mathrm{p}_{n} \quad$ dual variables associated with node $n$;

$\Phi_{X} \quad$ probability for method $X$;

fm multiplying factor;

$\eta_{X}(p c)$ performance metric for method $X$ to solve case $p c$;

$\eta^{*}(p c)$ best performance metric found with any method;

$p c \quad$ a computational case of the problem, where $p$ belongs to a representative set of cases.

\section{INTRODUCTION}

T HE power system generation scheduling is a crucial problem that evaluates the system future operation for pursuing the trade-off between economical and reliable use of power generation resources. Consequently, the scheduling model results from a mathematical representation of the system's components (reservoirs, power plants, transmission lines) and forecast for the operational conditions (weather, equipment failures, water inflow, demand). Considering that power systems can have different configurations, complexities, and sizes, the generation scheduling problem is usually solved by a set of different optimization models. In centralized-dispatch electricity markets [1], this task is executed by an Independent System Operator (ISO), which conducts studies ranging from the long/medium-term to short-term scheduling problems. The short-term include the Unit Commitment (UC) problem, which aims at finding the optimal generation schedule while meeting operational and system-wide constraints over a short-term horizon (typically 24 to 168 hours). Due to a fast-growing demand and increasing use of intermittent (renewable) production sources (wind and solar), ISOs are facing unprecedented challenges to maintain a reliable and economic operation; therefore, many works are focused on considering uncertainties in the UC (e.g., [2]-[4]). However, for some hydrothermal systems, the huge number of reservoirs, power plants, and transmission lines, precludes the ISO from considering the uncertainties associated with the system's operation. For instance, in the Brazilian case [5], which has the largest capacity for water storage and one of the largest transmission networks in the world, does not consider uncertainties in the UC problem. In Brazil, the power demand may deviate from predicted values due to several factors, such as the weather, equipment failures, among others. Further, about $53 \%$ of the hydro plants are run-of-river ones [6]; hence, the day-ahead operation is significantly affected by the water inflow uncertainties as well. Also, Brazilian's generation expansion planning predicts a high penetration of intermittent power plants [7] (wind, solar, and small hydroelectric), thereby further increasing the uncertainties for the-day ahead scheduling. All this justifies the interest in a Stochastic Hydrothermal Unit Commitment (SHUC) problem where uncertainty is related to water availability and demand. Besides the uncertainties, the SHUC pre- sents additional complexities such as nonlinear functions and binary variables to model a huge number of the system components and their constraints. Consequently, efficient strategies must be used to efficiently solve the SHUC problem. The main ones are mixed-integer programming, Lagrangian relaxation, Benders' decomposition, augmented Lagrangian, and heuristic approaches [2]. Due to the different characteristics, such as the hydro predominance, and the structure of the constraints, we apply decomposition approaches to managing the SHUC problem. Considering dualization approaches, the literature shows that for the stochastic UC the most common LR-based decomposition schemes are Scenario Decomposition (SD) [8] - [9] and Unit (or space) Decomposition (UD) [10] - [11]. The former split the problem into many sets of deterministic subproblems, while the latter decomposes the problem by physical characteristics (for instance, power plants). Some recent works present different techniques to solve the SHUC. The survey [12] describes the representation of uncertainty, different problem formulations, and the most common decomposition techniques applied to solve stochastic UC problems. Reference [13] proposes a strategy for the non-convex SHUC problem based on multi-horizon trees solved by a generalized Benders' decomposition. An extensive updated review [4] describes the main UC models and solution strategies, providing over 600 citations, many of which in the three recent years.

The main contributions of this paper are two-fold. On one hand, we propose a primal recovery (PR) strategy, based on a novel combination of Lagrangian Heuristics and Benders' decomposition approach [14] to generate primal feasible solutions. The PR is composed of the forward and backward steps in Benders' decomposition framework: the former uses the "convexified" primal solution [15] provided by the LR approach, while the latter iteratively improves the cost-to-go functions that evaluate the SUCH decisions in the next forward iteration. On the other hand, we assess the impact of the different decomposition strategies on the proposed PR strategy. Indeed, this paper provides improvements in the findings in [16], where four decompositions for the SHUC problem were compared in terms of computing time and quality of the lower bound. Here we analyze the performance of the two most promising decompositions (UD and SD) in terms of optimality gap, applying the (novel) PR technique for both schemes to perform a fair comparison.

This paper is organized as follows: in sections III and IV we describe the mathematical representation of the SHUC, and the two LR decomposition strategies employed to solve the problem. The proposed PR technique is presented in section V. Finally, in sections VI and VII, we show the computational results and state our conclusions, respectively.

\section{Two-Stage Stochastic Programming Problem}

We tackle the SHTUC problem of a risk-neutral Independent System Operator (ISO) in a tight-pool electricity market. Since there are several variants of this type of market, we use the Brazilian case, where the generating companies submit operating $\operatorname{costs}^{1}$ and availability data for the ISO, which then optimizes the day-ahead dispatch to meet the load at the minimum cost. The power load for each bus $\left(D_{b}\right)$ and inflows for

\footnotetext{
${ }^{1}$ In fact, only thermal plants submit fixed and variable costs. The hydro costs, given by an expected future cost function, is calculated also by ISO, which uses medium and long-term generation scheduling optimization models.
} 
each reservoir $\left(y_{r}\right)$ are discrete random parameters, and we use the well-known two-stage scenario-based approach for handling this uncertainty by a finite number of scenarios. Figure 1 illustrates a two-stage scenario tree with two realizations of inflow and load in the second stage. Each stage refers to three periods of time, representing decision nodes for the system operation. Consequently, the corresponding scenario tree has a total of 9 nodes.

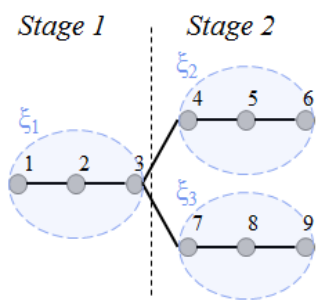

$$
\begin{aligned}
& \xi_{1} \subset\left\{D_{b 1}, D_{b 2}, D_{b 3}\right\} \cup\left\{y_{r 1}, y_{r 2}, y_{r 3}\right\} \\
& \xi_{2} \subset\left\{D_{b 4}, D_{b 5}, D_{b 6}\right\} \cup\left\{y_{r 4}, y_{r 5}, y_{r 6}\right\} \\
& \xi_{3} \subset\left\{D_{b 7}, D_{b 8}, D_{b 9}\right\} \cup\left\{y_{r 7}, y_{r 8}, y_{r 9}\right\} \\
& \forall b, r \\
& \text { Realization nodes } \\
& \text { Decision nodes }
\end{aligned}
$$

Figure 1. Illustration of the uncertain data.

The root node $n=1$ corresponds to the first period of the horizon. All the remaining nodes have an ascendant node and a set of descendant nodes. The ascending of node $n$ is termed $n_{-1}$ with the transition probability $p_{n / n_{-I}}$. The probabilities of the nodes are given recursively by $p_{1}=1$ and $p_{n}=p_{n / n_{-l}} p_{n_{-l}}, n>1$. The ascendant node of node $n$ at $t$ periods back in time is $n_{-t}$. The immediate descendants of node $n$ are $N_{+1}(n)$ and nodes with $N_{+1}(n)=\varnothing$ are called leaves. Moreover, the set of all the leaves of the tree is called $N_{L}$ and the set of all nodes of stage $h$ is denoted by $N^{h}$. The path from the root node to any leaf represents a scenario, indexed by $\omega$ and represented by the set $N_{\omega}$. The probability of each scenario is equal to the probability of the last node of the path, i.e., the leaf node. In the first stage, the realization $\xi_{1}$ is unique, while the second stage has many realizations of the random variables representing the uncertain demands. Since random variables are included via a scenario tree, it is possible to solve a deterministic equivalent (DE) program [17]. Then our two-stage SHTUC problem formulated via the DE program is stated by equations (1)-(14).

$$
\begin{aligned}
& \min \sum_{n=1}^{N} p_{n} \cdot \sum_{i=1}^{I}\left(F_{i n}+S C_{i} \cdot u p_{i n}\right) \\
& \text { s.t. : } v_{r n}-v_{r n_{-1}}+0,0036 \cdot\left[d_{r n}-\sum_{r^{+} \in R_{r}^{+}} d_{r^{+} n_{-\tau}}\right]=0,0036 \cdot y_{r n} \text {, } \\
& v_{r}^{\min } \leq v_{r n} \leq v_{r}^{\max }, v_{r n} \geq v_{r}^{\text {target }}, n \in N_{L}, d_{r n}=s_{r n}+\sum_{j=1}^{J_{r}} q_{j m}, s_{r m} \leq s_{r}^{\text {max }}, \\
& p h_{r n}=\sum_{j=1}^{J_{r}} p h g_{j m n}, p h g_{j m} \leq f p h g_{j m}^{c h}\left(v_{r m}, q_{j m}, d_{r m}, s_{r m}, z_{j m}\right), c h \in C H_{j r} \\
& p h g_{j r}^{\min } z_{j m} \leq p h g_{j m} \leq p h g_{j r}^{\max } z_{j m}, q_{j r}^{\min } z_{j m} \leq q_{j m} \leq q_{j r}^{\max } z_{j m}, \\
& z_{j r n} \in\{0,1\} \text {, } \\
& \sum_{r=1}^{R}\left(\sum_{j=1}^{J_{r}} p h g_{j r}^{\max } \cdot z_{j r n}-p h_{r n}\right) \geq \operatorname{Res}_{n} . \\
& F_{i n} \geq f o p t_{i n}^{c f}\left(p t_{i n}, u_{i n}\right), c f \in C F_{i}, u_{i n}-u_{i n_{-1}}=u p_{i n}-u d_{i n},
\end{aligned}
$$

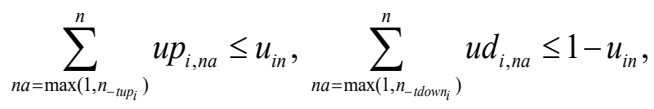

$$
\begin{aligned}
& p t_{i n}-p t_{i n_{-1}} \leq \Delta_{i}^{u p},-p t_{i n}+p t_{i n_{-1}} \leq \Delta_{i}^{d o w n}, 0 \leq p t_{i n} \leq p t_{i}^{\max }-p t_{i}^{\min },
\end{aligned}
$$

$$
\begin{array}{ll}
p t_{i n} \leq\left(p t_{i}^{\max }-p t_{i}^{\min }\right) \cdot\left(u_{i n}-u p_{i n}\right), & \forall t u p_{i}=0, \\
p t_{i n} \leq\left(p t_{i}^{\max }-p t_{i}^{\min }\right) \cdot\left(u_{i n}-u d_{i n_{+1}}\right), & \forall t u p_{i}=0, \\
p t_{i n} \leq\left(p t_{i}^{\max }-p t_{i}^{\min }\right) \cdot\left(u_{i n}-u p_{i n}-u d_{i n_{+1}}\right), & \forall t u p_{i} \geq 1, \\
u_{i n}, u p_{i n}, u d_{i n} \in\{0,1\}, & \\
\sum_{b=1}^{B} \sum_{r \in R_{b}} p h_{r n}+\sum_{b=1}^{B} \sum_{i \in I_{b}} p t_{i n}+u_{i n} \cdot p t_{i}^{\min }=\sum_{b=1}^{B} D_{b n}, & \\
-f l_{l}^{\max } \leq \sum_{b=1}^{B} \Gamma_{l b}\left(\sum_{r \in R_{b}} p h_{r n}+\sum_{i \in I_{b}} p t_{i n}+u_{i n} \cdot p t_{i}^{\min }-D_{b n}\right) \leq f l_{l}^{\max } .
\end{array}
$$

The objective function (1) is given by the expected value of two terms: the piecewise linear cost occurred in nominal operation, and a fixed start-up cost. The constraints (2)-(7) describe the reservoir and the hydro plants' operation. The operation of the thermal plants is represented by (8)-(12), while (13)-(14) are the classical DC transmission network constraints. Solving problem (1)-(14) directly with an off-the-shelf optimization solver is prohibitive due to its high complexity and the tight running-time requirements. Hence, decomposition techniques come into play to break (1)-(14) into smaller subproblems. The literature provides a range of decomposition approaches [4] and, in this work, as previously mentioned, we use LR. The LR solution strategy is based on decomposition schemes where the problem is divided into subproblems. For illustrate these subproblems, the constraints are structured as follows: constraints (2)-(3) compose the set $C_{r n}^{\mathrm{HA}}$; (4)-(7) compose the set $C_{r n}^{\mathrm{HE}} ;(8)-(12)$ compose the set $C_{i n}^{\mathrm{T}} ;(13)-(14)$ compose the set $C_{n}^{D}$; and (2)-(14) for $\forall n \in N(\omega)$ composing $C_{\omega}^{C}$, a set with all the constraints for a given scenario $\omega$. In this structure, the hydro modeling is more explored in the splitting approach, since, as presented in [16], its detailed representation requires more processing time when it is not properly divided. The problem (1)-(14) is solved in an LR fashion together with a PR heuristic, as depicted in Figure 2.

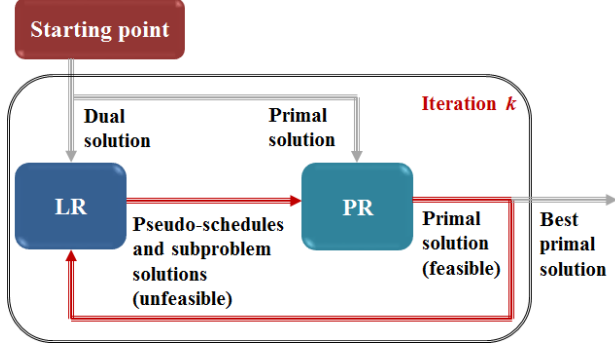

Figure 2. Strategy to solve the problem.

The strategy starts with an initial solution, which can be obtained by solving a simplified version of the problem or by the experience of the ISO. In our case, we use the primal and dual solution of the continuous relaxation of (1)-(14) that is relatively easy to obtain. At each iteration, an LR and a PR are executed simultaneously. The LR results (primal solution and pseudo-schedule ${ }^{2}$ ) of iteration $k$ are used as input for the PR strategy, whose primal feasible solution is used as a flat (all-0) eps-subgradient for the LR at iteration $k+1$. The LR is solved using a Bundle-type method [18], where the details can be ${ }^{2}$ A convex combination of the subproblems' solutions that is generated by the solution process in the LR. 
seen in [19] - [20].

On the other hand, the solution employed by the PR is based on heuristics that combine the solution of the subproblems with approximations of the cost-to-go function, typically employed in the context of Benders' decomposition. It is worth mentioning that the solution given by the LR step is a significant part of the algorithm and the kind of decomposition scheme applied influences the quality of its results. Then, the different decomposition schemes applied are described in Section 4, and the technique used in the PR is detailed in Section 5 .

\section{LR DECOMPOSITION STRATEGIES}

The problem (1)-(14) can be decomposed in different ways employing the LR technique. The most common approaches are the unit decomposition (UD) and the scenario decomposition (SD). In the former, the whole problem is separated by its physical characteristics, and in the latter, the problem is divided into many deterministic UC subproblems. The different strategies are illustrated in Figure 3.
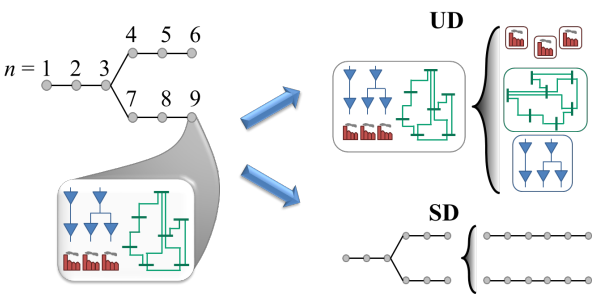

Figure 3. Illustration of unit and scenario decomposition schemes

The strategies are briefly described in the next Sections. For further details on the decomposition schemes and the technique used in the LR step see reference [16].

\section{A. Unit Decomposition}

This decomposition scheme separates the problem by its component characteristics: one subproblem per thermal plant, one subproblem for each node related to the transmission network, one subproblem with the reservoirs in the same chain, and one subproblem for each hydro plant in each node. Figure 4 illustrates the variable coupling between the subproblems of the UD, each circle represents a group of subproblems.

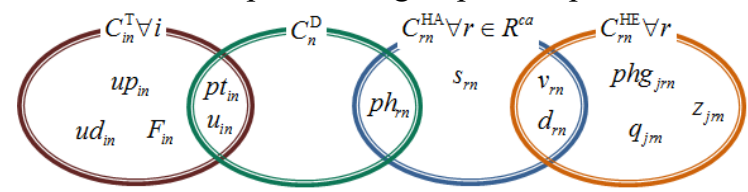

Figure 4. Illustration of the relationship between variables for UD.

To decompose the problem, we apply the variable splitting technique [21] and relax the spinning reserve constraints. The rationale of this strategy is to decompose as much as possible the constraints regarding reservoirs and hydro plants problems given its particular characteristics and considering the advantages presented in [16]. This strategy results in four groups of subproblems and a dual problem with $N \cdot(I+3 \cdot R+1)$ variables. The subsets $C_{r n}^{\mathrm{HA}}$ and $C_{n}^{D}$ result in $C A$ and $N$ Linear Programming (LP) problems, respectively. Furthermore, $C_{r n}^{\mathrm{HE}}$ and $C_{\text {in }}^{\mathrm{T}}$ produce $N \cdot R$ and $I$ MILP problems, respectively.

\section{B. Scenario Decomposition}

In this scheme, all the constraints belonging to nodes of the scenario $\omega, N(\omega)$, make up the set of constraints $C_{\omega}^{C} C_{\omega}^{\mathrm{C}}$.
Figure 5 illustrates the sets of constraints for the problem of Figure 3, representing the variables that couples the subproblems. The set $N^{1}$ represents all the nodes of stage 1 .

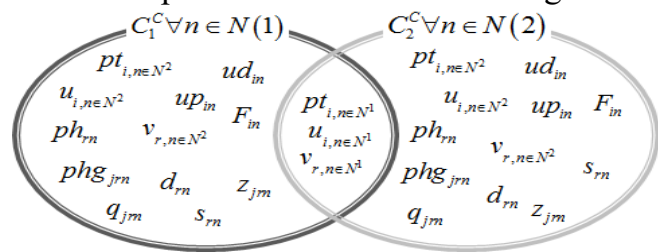

Figure 5. Illustration of the relationship between variables for SD.

Consequently, to decouple the problem we apply the variable splitting technique in the linking variables of the first stage (non-anticipativity constraints), separating it in single-scenario deterministic subproblems. This strategy results in $\Omega$ MILP subproblems and a dual problem with $N S^{1} \cdot \Omega \cdot(2 \cdot I+R)$, where $N S^{1}$ is the number of nodes in the first stage.

\section{Primal Recovery Technique}

The application of the LR in nonconvex problems usually does not result in a feasible solution. However, it gives a dual solution and a lower bound for the optimal solution. The LR does not solve a nonconvex problem; instead, it solves a convexified version of the problem [22]-[24]. For MILP problems, it means the solution is feasible for all the constraints, less the integrality ones, resulting in a solution called pseudoschedule, which is a convex combination of the subproblems' solutions. The pseudo-schedule solution can be used with the results of the LR-subproblems as heuristic [25] - [26] to recover a primal feasible solution. Note that the Bundle method can exploit the primal feasible solution found by the heuristic as part of the model: indeed, the value of any feasible solution is an upper bound on the maximum of the Lagrangian dual, that can be incorporated in the model (as a "flat", all-0, epsilonsubgradient) used to determine the next iterate, thereby improving the LR performances.

\section{A. Forward step}

The forward step follows a strategy successfully used in the different contexts of the mostly-thermal deterministic UC [25]: exploiting both the continuous feasible pseudo-schedule and integer unfeasible solution of the LR-subproblems at each iteration. Although the solution given by the LR-subproblems is feasible for its subproblem, they are not for the original problem. While in the simpler context of [25] this was done by a simple constructive heuristic, here proximal terms are added to the original objective function in the PR step. However, similarly to [25], the idea is to perform a sliding window approach as illustrated in Figure 6.
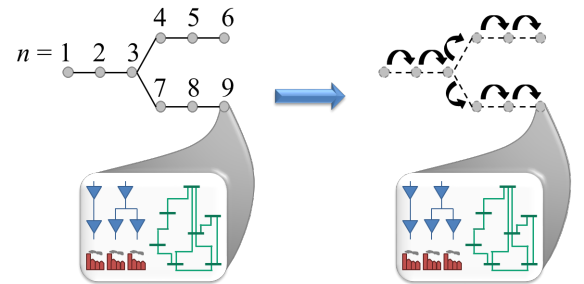

Figure 6. Illustration of the decomposition in the PR step.

As illustrated instead of solving one multi-period problem, a sequence of single-period smaller subproblems (PRsubproblems) are solved in sequence, from the first period to the last one, using the solution of the previous subproblem as 
input to the current one. The typical issue with these approaches is their myopic nature: decisions taken in the initial steps do not consider the constraints in later ones. In our SHUC, this would easily yield infeasibilities due to, mainly, too little water being left for the last periods. To improve on this, we use the LR solutions (both the convexified and the integer one) as reference points that the subproblems should aim at reaching. That is, in each subproblem related to the node $n$, the original objective function, oof, is modified with the introduction of a proximal term, $p x t$, weighted by a parameter, as follows:

$$
\min \mu_{1} \cdot \text { oof }_{n}+\left(1-\mu_{1}\right) \cdot \text { pxt }_{n} \text {, }
$$

subject to: (2)-(14) for a specific node $n$.

The role of the proximal term is to reduce the distance to the available solutions (pseudo-schedule and LR solution). In particular, the proximal term has two components, one related to the pseudo-schedule and another regarding the LR solution, as shown below.

$$
\begin{aligned}
& p x t_{n}=\mu_{2} \cdot p x t_{n}^{P S}+\left(1-\mu_{2}\right) \cdot p x t_{n}^{L R,} \\
& p x t_{n}^{P S}=\mu_{3} \cdot\left\|x_{n}-\tilde{x}_{n}\right\|+\left(1-\mu_{3}\right) \cdot\left\|u_{n}-\tilde{u}_{n}\right\|, \\
& p x t_{n}^{L R}=\left(1-\mu_{3}\right) \cdot\left\|x_{n}-\hat{x}_{n}\right\|+\mu_{3} \cdot\left\|u_{n}-\hat{u}_{n}\right\| .
\end{aligned}
$$

All the terms in (16) are scaled by an estimate of the maximum values that they can take, to avoid numerical difficulties and to put all the terms at the same magnitude (save for the appropriate choice of the weight parameters that are performed experimentally). While the original approach in [25] was enough to obtain good solutions for a mainly thermal UC, this was not always the case here. The issues are the higher complexity of the system due to the stochastic nature of the problem, and the reliance on hydro production that made it crucial to store enough water for the last stages. The "memory" provided by the LR solutions is not always sufficient to attain this. For this reason, the heuristic has been improved by adding another step em the PR strategy, where we consider approximations of the Benders' optimality cuts to the subproblems, as described next.

\section{B. Backward step}

The PR strategy can be improved using a backward recursion, where an approximation of the cost-to-go function is added to each RP-subproblem. This function measures the consequence of the decisions in future periods, and are build based on Benders' decomposition method [14]. We remark that Benders' decomposition requires convexity to assure the convergence of the approach; however, in our case, this is automatically obtained because the LR solves a convexified version of the original problem. This approximation does not guarantee the optimality of the decision, but still provide useful information (a lower bound) on the future cost of each decision, significantly improving the performances of the heuristic in practice. We present below the formulation of the node $n$ subproblem related to the backward in the PR strategy. Note that the proximal terms are not considered $\left(\mu_{1}=1\right)$; furthermore, an approximation of the cost-to-go-function, $a f c$, is added to the objective function.

$$
\begin{aligned}
& \min \operatorname{oof}_{n}+a f c_{n}, \\
& \text { s.t.: (2)-(5), (7)-(11), (13)-(14), } \\
& 0 \leq z_{j r n}, u_{i n}, u p_{i n}, u d_{i n} \leq 1, a f c_{n}+\pi_{n+1}^{\prime} \cdot\left[x_{n} \mid u_{n}\right] \geq f c_{n+1} .
\end{aligned}
$$

In turn, $a f c$ is a convex polyhedral function represented by linear constraints as standard in Benders' decomposition. Subproblems in the backward step use the state variables obtained in the forward step to recursively build the linear approximations. On the other hand, the set of linear approximations of the cost to go function are used in all the next iteration of the forward step, as illustrated in Figure 7.

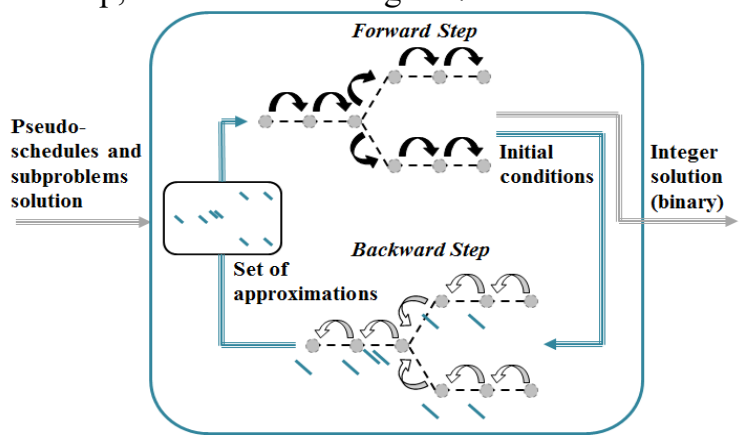

Figure 7. Interaction of the forward and backward steps in the PR strategy.

\section{Algorithm}

Besides the two aforementioned steps, at the end of each iteration, a full economic dispatch (LP problem (1)-(5), (7)-(11), (13) and (14) with binary variables $z, u, u p$ and $u d$ fixed by the forward step) is solved. A complete iteration of the PR phase is illustrated in Figure 8.

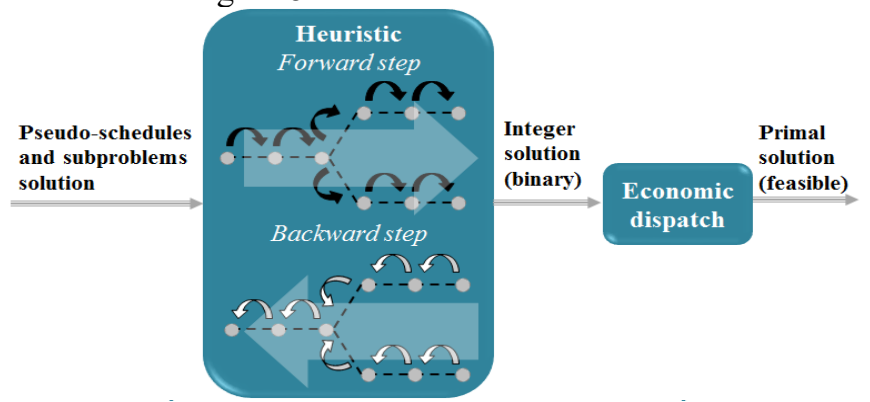

Figure 8. One iteration of the PR strategy.

All the cost-to-go approximations added in one iteration are kept for all the next iterations since they are valid lower bounds for all the subproblem solutions. As illustrated in Figure 1, both LR and PR are performed at each iteration, and this procedure finishes by Bundle's stopping criteria or the time limit. The best primal solution found by the algorithm is the solution of the SHTUC problem.

\section{RESULTS}

The results have been obtained on an Intel Xeon CPU X5690 (3.47 GHz) computer with 32 GigaBytes of RAM. The LP and MILP problems are solved using a general-purpose optimization solver. The different approaches are compared utilizing usual statistical metrics (average values and standard deviation), as well as employing the performance profile technique [27]. The performance profiles are cumulative distribution functions for a given metric (number of iterations, processing time, objective function, etc.), which depend on the multiplying factor, i.e., is the relation between the current method and the best one. The expression of the performance profile is given by:

$\Phi_{X}(f m)=\frac{\text { number of cases which } \eta_{X}(p c) \leq f m \cdot \eta^{*}(p c)}{\text { total of evaluated cases }}$,

The solution strategy is assessed for the hydrothermal sys- 
tems showed in Table 1. The systems have different initial conditions and scenarios trees to produce a wide range of results.

\begin{tabular}{c|c|c|c|c|c|c}
\multicolumn{6}{c}{ Table 1. Test Systems for Hydrothermal SHUC Problems. } \\
\hline System & \multicolumn{2}{|c|}{$\begin{array}{c}\text { Number of } \\
\text { plants }\end{array}$} & \multicolumn{2}{c|}{$\begin{array}{c}\text { Generation } \\
\text { capacity (\%) }\end{array}$} & $\begin{array}{c}\text { Generation } \\
\text { capacity (MW) }\end{array}$ & $\begin{array}{c}\text { Storage } \\
\text { capacity (hm }\end{array}$ \\
& H & T & H & T & & \\
A & 7 & 14 & 25.0 & 75.0 & $21,297.5$ & $5,635.1$ \\
B & 7 & 14 & 75.0 & 25.0 & $9,224.0$ & $9,309.0$ \\
C & 10 & 10 & 50.0 & 50.0 & $16,132.2$ & $10,737.5$ \\
D & 14 & 7 & 76.3 & 23.7 & $16,046.5$ & $14,944.1$ \\
E & 14 & 7 & 25.2 & 74.8 & $9,671.0$ & $5,507.2$ \\
DD & 28 & 15 & 74.8 & 25.2 & $29,922.6$ & $44,043.99$ \\
\hline
\end{tabular}

$\mathrm{H}$ stands for hydro and T for thermal power plants.

Furthermore, the UD and SD schemes used in LR are compared using the optimality gap $=(U B-B U B) / B U B$, where the upper bound $U B$ is the solution given by some strategy and $B U B$ is the best known upper bound for all the strategies tested. Among the strategies, we also have solved the deterministic equivalent through the same general-purpose MILP solver used in the decomposition schemes.

\section{A. Systems A to E}

Test systems A to E have the same power system (46 buses and 95 transmission lines), but the location in the electrical network and the number of power plants are different. The electrical system A is illustrated in Figure 9.

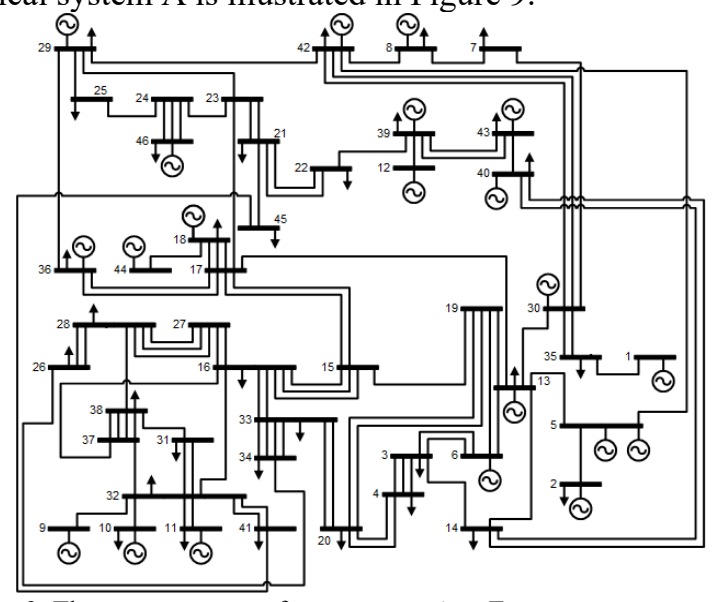

Figure 9. The power system of test systems A to E.

The five test systems are assessed with four scenario tree sizes and three initial conditions, which results in 60 instances. The results are first presented in Figure 10 and Figure 11, using the performance profile approach for processing time and optimal solution, respectively.

Table 2 complements the result, where we present the average values (and standard deviation, in brackets) of the gap, processing time, oracle time (time to solve the LR subproblems), PR time (total time spend solving the PR strategy) and the number of iterations for all approaches. All the 60 instances for the MILP (i.e., DE approach) stopped by the time limit ${ }^{3}$ (40 minutes). Also, $75 \%$ of the LR decomposition schemes did not reach the convergence within this time. Only 12 and 11 instances stopped before 40 minutes for the UD and SD, respectively. For the SD scheme the instances stopped pretty close to the time limit, meanwhile for the former (UD), we have some variability of the processing time, as shown in Table 2 .

\footnotetext{
${ }^{3}$ Some instances take some extra time since the time limit was reached in the middle of the solver processing.
}

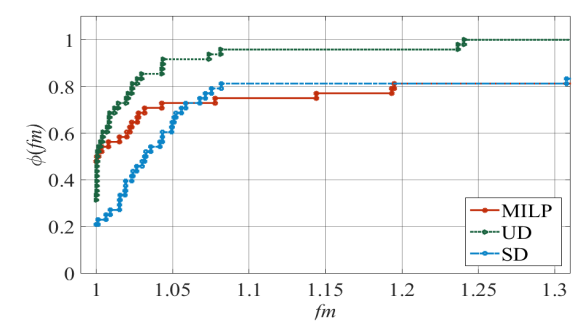

Figure 10. Performance profile for the processing time.

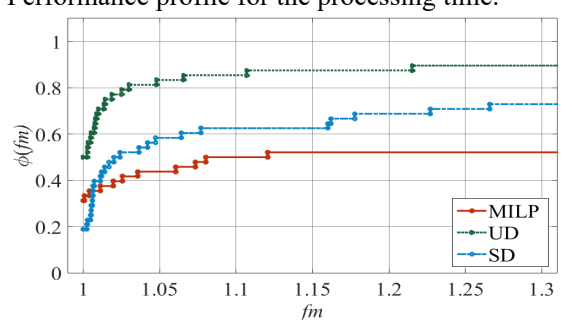

Figure 11. Performance profile for the optimal solution. Table 2. Results for Test Systems A-E.

\begin{tabular}{l|c|c|c|c|c}
\hline Strategy & Gap [\%] & Time & Oracle time [\%] & PR time [\%] & Iterations \\
\hline \multirow{2}{*}{ MILP } & $1,134.50$ & 1.05 & - & - & - \\
& $(2,194.87)$ & $(0.0)$ & - & - & - \\
UD & 20.11 & 1 & 11.48 & 18.87 & 3.58 \\
& $(58.41)$ & $(0.19)$ & $(8.74)$ & $(11.63)$ & $(4.33)$ \\
SD & 188.89 & 1.08 & 94.23 & 5.39 & 1.0 \\
& $(1,011.49)$ & $(0.06)$ & $(4.36)$ & $(4.07)$ & $(1.08)$ \\
\hline
\end{tabular}

The time and the number of iterations is relative to the smaller values, i.e., UD for the time and SD for the iterations: Time $=1$ corresponds to $38.1 \mathrm{~min}$, Iteration $=1$ corresponds to 13.2 iterations.

As can be seen, the primal recovery strategy with UD and SD schemes have better performance than solving via a single MILP model. UD scheme has a smaller average processing time compared to the other strategies, followed by MILP and $\mathrm{SD}$, as presented in Table 2. Considering a tolerance of $5 \%$ in the optimal solution $(f m=1.05)$, we have about $82 \%$ of the cases solved by UD, $58 \%$ solved by SD and $43 \%$ solved by MILP. Table II also reports a substantial difference in the average gap between the strategies. We also compare the strategies regarding the size of the scenario tree. The idea is to observe the performance of the approaches comparing the upper and lower bounds given by each method. We perform this comparison using the optimality gap and the lower bound $l b$ gap $=(L B-B L B) / B L B$, where $L B$ is the lower bound given by some strategy and $B L B$ is the best known lower bound for all the strategies tested. Figures Figure 12 and Figure 13 show the gap and $l b \_g a p$ for the solution strategies, respectively.

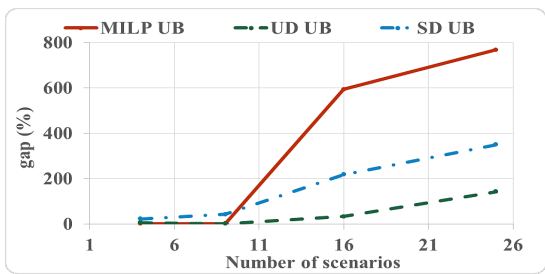

Figure 12. Performance of upper bounds versus the number of scenarios.

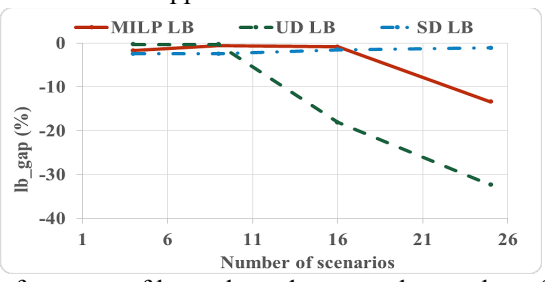

Figure 13. Performance of lower bounds versus the number of scenarios. 
In general, the UD scheme presents a smaller optimality gap, but with the poorest lower bounds. SD provides much better lower bounds, but a solution of somewhat lesser quality. However, our PR strategy dramatically outperforms solving the entire problem as a MILP with 16 scenarios or more.

Finally, we can examine in detail some primal results to compare the different configurations of power plants. Figure 14 shows the results for test systems A (firsts graphs), B (second graphs), and C (last graphs) for the problem with 25 scenarios solved by the three strategies. The curves on the left (red ones) represent the solution with MILP, the curves on the middle (green ones) the solution with UD, and the curves on the right (blue ones) the solution given by the SD scheme. We remark that there is a deficit of energy in the MILP solution, meaning it is more way expensive than the others; in other words, the solver could not deliver a reasonable solution in the given time. Furthermore, in system $\mathrm{A}$, the thermal power plants follow the variations of the demand. This happens also in system $\mathrm{E}$ since thermal plants are predominant. On the other hand, in test systems with a hydro predominance (B and D), thermal generators behave as base-load power plants.

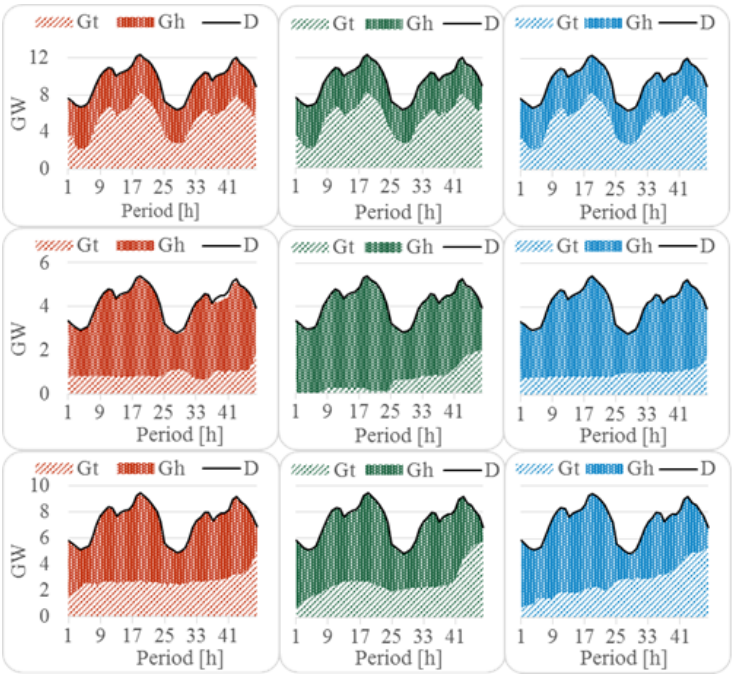

Figure 14. Power production and demand over two days for a problem with 25 scenarios (Gt, Gh, and D stand for thermal generation, hydro generation, and power demand, respectively).

\section{B. System DD}

In this section, it is compared the strategies for system DD, which is an expansion of test system $\mathrm{D}$ to an 82-buses power system with 143 transmission lines. We consider three initial conditions and six different scenario trees (with four and nine scenarios and three different realizations for each one), resulting in 18 cases for the same system. Figure 15 shows the performance profiles for the optimal solution for a given time limit of 120 minutes; all the strategies always hit the time limit, which is expected considering the size of the problem.

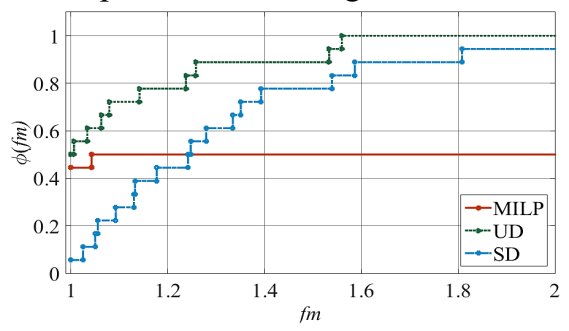

Figure 15. Performance profile for the optimal solution - system DD.
The performance profile shows that our primal recovery strategy with UD scheme has a better performance in most of the instances. Considering a tolerance of $20 \%$ in the optimal value, $f m=1.2$, about $78 \%$ of the instances are solved by UD, $50 \%$ by the MILP, and $44 \%$ by the SD. We present the average values (and standard deviation) in Table 3.

Table 3. Results for Test System DD.

\begin{tabular}{|c|c|c|c|c|}
\hline Strategy & Gap [\%] & Oracle Time [\%] & PR Time [\%] & Iterations \\
\hline \multicolumn{5}{|c|}{ 4 Scenarios } \\
\hline MILP & $0.47(1.34)$ & - & - & - \\
\hline UD & $0.55(20.23)$ & $29.22(14.22)$ & $27.74(3.62)$ & $30.0(17.1)$ \\
\hline SD & 7.47 (55.93) & $97.98(0.66)$ & $2.01(0.65)$ & $1.0(0.4)$ \\
\hline \multicolumn{5}{|c|}{9 Scenarios } \\
\hline MILP & $\begin{array}{c}13,164.1 \\
(11,369.2)\end{array}$ & - & - & - \\
\hline UD & 0.69 (1.96) & $19.84(14.81)$ & $25.42(3.53)$ & $24.6(24.3)$ \\
\hline SD & $4.58(12.60)$ & $98.10(0.56)$ & $1.89(0.56)$ & $1.0(0.3)$ \\
\hline
\end{tabular}

The value within brackets represents the standard deviation. The number of iterations is relative to the smaller values, i.e., these for SD: Iteration $=1$ corresponds to 4.9 and 3.6 iterations for 4 and 9 scenarios, respectively.

As in the previous section, the decomposition schemes are more and more competitive to the MILP as the size of the scenario tree grows. Regarding the comparison between the two decomposition schemes, in UD the PR and LR subproblems take the same relative time, while in SD most of the time is spent to solve LR subproblems. The number of iterations in $\mathrm{SD}$ is smaller, which may be a factor in the poorer performance of the PR approach: fewer iterations mean fewer approximations of the cost-to-go function, and hence possibly poorer information for the PR strategy. Nevertheless, even the SD scheme results in better gaps than the problem solved as a MILP model.

\section{Deterministic Results}

Currently, the day-ahead model used to obtain the generation scheduling in Brazil is deterministic and solved using a single MILP problem. Hence, we found it useful to test the performance of decomposition strategies (clearly UD, since there are no scenarios to decompose by). For this purpose, we developed and solved a very large deterministic UC. The power system solved has 164 hydroelectric and 412 thermoelectric power plants; due to memory limitations, the transmission system constraints were disregarded. The results for five different cases (different curves of demand and water inflow) are shown in Table 4.

Table 4. Results for a Bigger System.

\begin{tabular}{c|c|c|c|c|c}
\hline Strategy & Case & LB (R\$) & UB (R\$) & Gap $^{\mathbf{4}}[\mathbf{\%}]$ & Time [min.] \\
\hline \multirow{5}{*}{ MILP } & 1 & $6,048,832$ & $40,197,464,370$ & 136,036 & 60 \\
& 2 & $5,542,550$ & $34,757,581,818$ & 139,358 & 60 \\
& 3 & $7,228,961$ & $39,378,674,530$ & 144,199 & 60 \\
& 4 & $6,512,480$ & $62,741,817,027$ & 323,724 & 60 \\
& 5 & $3,889,464$ & $17,239,231,489$ & 174,507 & 60 \\
\hline \multirow{5}{*}{ UD } & 1 & $3,950,691$ & $29,642,903$ & 0.0 & 60 \\
& 2 & $3,647,832$ & $25,086,238$ & 0.0 & 60 \\
& 3 & $4,709,265$ & $27,383,828$ & 0.0 & 60 \\
& 4 & $4,118,128$ & $19,497,174$ & 0.0 & 60 \\
& 5 & $3,173,259$ & $9,978,269$ & 0.0 & 60 \\
\hline
\end{tabular}

The table shows that even in a deterministic problem (close to the Brazilian electrical system) decomposition strategies with our new PR scheme are highly competitive with directly solving the problem with a MILP solver.

\footnotetext{
${ }^{4}$ This value is not the relative difference between LB and UB for each strategy, but the optimality gap as defined in section VI.
} 


\section{CONCLUSIONS}

Lagrangian Relaxation is a known decomposition technique that exploits the structure of the problem, splitting it into many subproblems. Its main drawback is its inability to produce feasible solutions to nonconvex problems. Therefore, it has to be complemented by other strategies to recover the primal feasibility, like inexact augmented Lagrangian [28] and heuristics [29]-[34]. In this work, we have proposed a novel PR scheme based on a combination of the solutions (both integer and continuous) given by LR-subproblems and Benders' approach. Our PR scheme is general and can be applied to the many different decompositions that SHUC can have. We tested it on the two main ones, unit and scenarios schemes, assessing its performances for a plurality of hydrothermal systems, up to a very large one based on the Brazilian power system. Our results show that decomposition strategies are competitive with solving the problem through a MILP solver. This is true already for the deterministic case, and the advantage grows as the problem's size (number of power plants or scenarios) does. Among the decomposition strategies, UD provides better solutions in less time, although SD provides better lower bounds. The latter result was already known [16], but this work shows the importance of considering all elements of a decomposition technique (LR and PR) together to choose the right decomposition approach.

\section{REFERENCES}

[1] L. A. Barroso, T. H. Cavalcanti, P. Giesbertz, and K. Purchala, "Classification of electricity market models worldwide," in International Symposium CIGRE/IEEE PES, 2005., Oct. 2005, pp. 9-16, doi: 10.1109/CIGRE.2005.1532720.

[2] M. Tahanan, W. van Ackooij, A. Frangioni, and F. Lacalandra, "Largescale Unit Commitment under uncertainty," 4OR, vol. 13, no. 2, pp. 115-171, Jan. 2015, doi: 10.1007/s10288-014-0279-y.

[3] Q. P. Zheng, J. Wang, and A. L. Liu, "Stochastic Optimization for Unit Commitment: A Review," IEEE Trans. Power Syst., vol. 30, no. 4, pp. 1913-1924, Jul. 2015, doi: 10.1109/TPWRS.2014.2355204.

[4] W. Ackooij, I. D. Lopez, A. Frangioni, F. Lacalandra, and M. Tahanan, "Large-scale unit commitment under uncertainty: an updated literature survey," Ann. Oper. Res., vol. 271, no. 1, pp. 11-85, Sep. 2018, doi: 10.1007/s10479-018-3003-z.

[5] T. N. Santos, A. L. Diniz, C. H. Saboia, R. N. Cabral, and L. F. Cerqueira, "Hourly Pricing and Day-Ahead Dispatch Setting in Brazil: The DESSEM Model," p. 8, 2020.

[6] Operador Nacional do Sistema Elétrico - ONS, "Diagrama Esquemático das Usinas Hidroelétricas do SIN," 2020. [Online]. Available: http://www.ons.org.br/paginas/sobre-o-sin/mapas.

[7] Ministério de Minas e Energia - MME, "Plano Decenal de Expansão de Energia 2029," 2020.

[8] S. Takriti, J. R. Birge, and E. Long, "A stochastic model for the unit commitment problem," Power Syst. IEEE Trans. On, vol. 11, no. 3, pp. $1497-1508,1996$.

[9] C. C. Carøe and R. Schultz, "A Two-Stage Stochastic Program for Unit Commitment under Uncertainty in a Hydro-Thermal Power System.," Konrad-Zuse-Zentrum für Informationstechnik Berlin, Berlin, Germany, 1997.

[10] M. Nowak and W. Römisch, "Stochastic Lagrangian Relaxation Applied to Power Scheduling in a Hydro-Thermal System under Uncertainty," Ann. Oper. Res., vol. 100, no. 1-4, pp. 251-272, Dec. 2000, doi: 10.1023/a:1019248506301.

[11] R. Nürnberg and W. Römisch, "A Two-Stage Planning Model for Power Scheduling in a Hydro-Thermal System Under Uncertainty," Optim. Eng., vol. 3, no. 4, pp. 355-378, Dec. 2002, doi: 10.1023/a: 1021531823935.

[12] M. Håberg, "Fundamentals and recent developments in stochastic unit commitment," Int. J. Electr. Power Energy Syst., vol. 109, pp. 38-48, Jul. 2019, doi: 10.1016/j.ijepes.2019.01.037.
[13] "Stochastic hydro-thermal unit commitment via multi-level scenario trees and bundle regularization | SpringerLink." https://link.springer.com/article/10.1007/s11081-019-09448-z (accessed Apr. 11, 2020).

[14] J. F. Benders, "Partitioning procedures for solving mixed-variables programming problems," Numer. Math., vol. 4, pp. 238-252, 1962.

[15] M. Guignard, "Lagrangean Relaxation," Top - J. Span. Soc. Stat. Oper. Res., vol. 11, no. 2, pp. 151-228, 2003.

[16] M. R. Scuzziato, E. C. Finardi, and A. Frangioni, "Comparing Spatial and Scenario Decomposition for Stochastic Hydrothermal Unit Commitment Problems," IEEE Trans. Sustain. Energy, vol. 9, no. 3, pp. 1307-1317, Jul. 2018, doi: 10.1109/TSTE.2017.2781908.

[17] J. R. Birge and F. Louveaux, Introduction to Stochastic Programming, 2nd ed. 2011.

[18] A. Frangioni, "Standard Bundle Methods: Untrusted Models and Duality," in Numerical Nonsmooth Optimization: State of the Art Algorithms, A. M. Bagirov, M. Gaudioso, N. Karmitsa, M. M. Mäkelä, and S. Taheri, Eds. Cham: Springer International Publishing, 2020, pp. 61-116.

[19] A. Frangioni, "Generalized Bundle Methods," SIAM J. Optim., vol. 13, no. 1, pp. 117-156, 2002, doi: 10.1137/S1052623498342186.

[20] A. Frangioni and E. Gorgone, "Bundle methods for sum-functions with 'easy' components: applications to multicommodity network design," Math. Program., vol. 145, no. 1-2, pp. 133-161, Jun. 2014, doi: 10.1007/s10107-013-0642-3

[21] O. Nilsson and D. Sjelvgren, "Hydro unit start-up costs and their impact on the short term scheduling strategies of Swedish power producers," Power Syst. IEEE Trans. On, vol. 12, no. 1, pp. 38-44, 1997.

[22] S. Feltenmark and K. C. Kiwiel, "Dual applications of proximal bundle methods, including Lagrangian relaxation of nonconvex problems," 1998.

[23] A. Frangioni, "About Lagrangian Methods in Integer Optimization," Ann. Oper. Res., vol. 139, no. 1, pp. 163-193, Oct. 2005, doi: 10.1007/s10479-005-3447-9.[24] C. Lemaréchal, "Lagrangian Relaxation," in Computational Combinatorial Optimization, M. Jünger and D. Naddef, Eds. Springer Berlin Heidelberg, 2001, pp. 112-156.

[25] A. Frangioni, C. Gentile, and F. Lacalandra, "Solving unit commitment problems with general ramp constraints," Int. J. Electr. Power Energy Syst., vol. 30, no. 5, pp. 316-326, 2008, doi: 10.1016/j.ijepes.2007.10.003.

[26] C. Sagastizábal, "Divide to conquer: decomposition methods for energy optimization," Math. Program., vol. 134, no. 1, pp. 187-222, Jun. 2012, doi: 10.1007/s10107-012-0570-7.

[27] E. D. Dolan and J. J. Moré, "Benchmarking optimization software with performance profiles," Math. Program., vol. 91, no. 2, pp. 201-213, Jan. 2002, doi: 10.1007/s101070100263.

[28] A. Belloni, A. L. D. Lima, M. E. P. Maceira, and C. A. Sagastizábal, "Bundle Relaxation and Primal Recovery in Unit Commitment Problems. The Brazilian Case," Ann. Oper. Res., vol. 120, no. 1-4, pp. 2144, Apr. 2003, doi: 10.1023/a:1023314026477.

[29] A. Daniilidis and C. Lemaréchal, "On a primal-proximal heuristic in discrete optimization," Math. Program., vol. 104, no. 1, pp. 105-128, Mar. 2005, doi: 10.1007/s10107-004-0571-2.

[30] L. Dubost, R. Gonzalez, and C. Lemaréchal, "A primal-proximal heuristic applied to the French Unit-commitment problem," Math. Program., vol. 104, no. 1, pp. 129-151, 2005, doi: 10.1007/s10107-005-0593-4.

[31] A. Borghetti et al., "Lagrangian relaxation and Tabu Search approaches for the unit commitment problem," in 2001 IEEE Porto Power Tech Proceedings (Cat. No.01EX502), Porto, Portugal, 2001, vol. vol.3, p. 7, doi: 10.1109/PTC.2001.964914.

[32] A. Diabat, O. Battaïa, and D. Nazzal, "An improved Lagrangian relaxation-based heuristic for a joint location-inventory problem," Comput. Oper. Res., vol. 61, pp. 170-178, Sep. 2015, doi: 10.1016/j.cor.2014.03.006.

[33] J. Tang, C. Yan, X. Wang, and C. Zeng, "Using Lagrangian Relaxation Decomposition With Heuristic to Integrate the Decisions of Cell Formation and Parts Scheduling Considering Intercell Moves," IEEE Trans. Autom. Sci. Eng., vol. 11, no. 4, pp. 1110-1121, Oct. 2014, doi: 10.1109/TASE.2014.2325860

[34] A. Mohammad Nezhad, H. Manzour, and S. Salhi, "Lagrangian relaxation heuristics for the uncapacitated single-source multi-product facility location problem," Int. J. Prod. Econ., vol. 145, no. 2, pp. 713-723, Oct. 2013, doi: 10.1016/j.ijpe.2013.06.001. 